\title{
A Novel Approach to High Resolution Fetal Brain MR Imaging
}

\author{
F. Rousseau ${ }^{1}$, O. Glenn ${ }^{1}$, B. Iordanova ${ }^{1,2}$, C. Rodriguez-Carranza ${ }^{1,2}$, \\ D. Vigneron ${ }^{1}$, J. Barkovich ${ }^{1}$, and C. Studholme ${ }^{1}$ \\ 1 University of California, San Francisco \\ 2 NCIRE, San Francisco
}

\begin{abstract}
This paper describes a novel approach to forming high resolution MR images of the human fetal brain. It addresses the key problem of motion of the fetus by proposing a registration refined compounding of multiple sets of orthogonal fast 2D MRI slices, that are currently acquired for clinical studies, into a single high resolution MRI volume. A robust multi-resolution slice alignment is applied iteratively to the data to correct motion of the fetus that occurs between $2 \mathrm{D}$ acquisitions. This is combined with an intensity correction step and a super resolution reconstruction step, to form a single high isotropic resolution volume of the fetal brain. Experimental validation on synthetic image data with known motion types and underlying anatomy, together with retrospective application to sets of clinical acquisitions are included. Results indicate the method promises a unique route to acquiring high resolution MRI of the fetal brain in vivo allowing comparable quality to that of neonatal MRI. Such data is highly valuable in allowing a clinically applicable window into the process of normal and abnormal brain development.
\end{abstract}

\section{Introduction}

Ultrasound is the modality of choice for clinical evaluation of the developing fetus. However, clinical diagnosis with ultrasound is sometimes unclear and in these cases, alternative studies with magnetic resonance imaging (MRI) can be essential [6, 4]. One such critical application, which is the focus of this paper, is in the evaluation of isolated ventriculomegaly, which despite the absence of other clinical findings, can be associated with neurodevelopmental disabilities in childhood and infancy.

The development of ultrafast 2D acquisition sequences has led to significant improvements in the clinical utility of fetal MRI ([10]). However, the slice acquisition time is still very critical and has to be as short as possible to reduce the impact of fetal motion on the exam, since fetal MRI is performed without sedation. As a result, sets of thick $2 \mathrm{D}$ slices are generally acquired in clinical studies, with motion commonly occurring between slices. Overall, the resulting image data is limited in its geometric integrity between slices due to motion, and in its through plane spatial resolution. In addition, body coils used in the imaging processes do not provide a homogeneous sensitivity over the field of view and, because of motion during the acquisition, can produce different a distortion profile over time (see figure 2). 
This work is motivated by the observation that current clinical imaging protocols make use of multiple orthogonal 2D multi-planar acquisitions to study the fetal brain. However, because of the motion between the slices, interpretation is limited to visual inspection by a trained radiologist, and does not allow direct quantitative measurements to be made on the 3D anatomy. The aim of this work is to develop and apply registration based reconstruction methods to the problem of correcting motion and intensity distortions between the different $2 \mathrm{D}$ acquisitions, and to then reconstruct a single higher resolution MRI image of the fetal brain.

As far as we know, this problem has not been discussed before in the literature. Moore et al. 8] built a high resolution dynamic heart model from isotropic coronal slices. The misalignment was corrected by registering a volume with sagittal and axial scout images. Image intensities were averaged together to generate a high resolution volume. Lötjönen et al. 7] have proposed an algorithm to correct translation-only movement artifacts simultaneously from short- and long-axis cardiac MR series. Locations of short-axis slices were optimized based on data from long-axis slices and vice versa.

In this article we report our work on this challenging problem and describe a fully automatic and accurate algorithm to correct slice misalignments, correct intensity distortions, and reconstruct a single high resolution image from sets of clinically typical low resolution fetal MRI data.

\section{Method}

The resolution of the low resolution source data is typically $1 \mathrm{x} 1 \mathrm{~mm}$ in plane with $3 \mathrm{~mm}$ thick slices. Multiple sets consisting of between 30 and 40 slices each are acquired over a period of around 20 seconds for each set. The slices are commonly acquired in an interleaved pattern (usually alternating odd-even) and significant movement of a centimeter or more can occur during the acquisition of one set of slices. Sets of slices are typically acquired in approximately orthogonal axes (coronal, sagittal and axial), providing complementary resolution. It is reasonable (and is confirmed visually for most slices) to assume that motion within the time period of one slice is negligible. We are posed the task of recovering the local relative alignment of each slice in 3 dimensions, but can make use of the consistent geometry provided in the through plane direction of a slice that is provided by the other orthogonal sets of slice data, to constrain the collective alignment of the multiple low resolution images.

Notations. Let us consider $n$ low resolution $3 \mathrm{D}$ images, denoted by $I_{L R}^{i}, i \in$ $\{1 \ldots n\}$. A so-called low resolution $3 \mathrm{D}$ image is a stack of $2 \mathrm{D}$ thick slices. The reconstructed high resolution $3 \mathrm{D}$ image is denoted by $I_{H R}$.

\subsection{Motion Correction}

In order to correct fetal motion, we propose to use a registration based method. Registration methods are usually classified as being either sparse (landmark) 
or dense (voxel) based. In the case of fetal brain MRI registration, because of the $3 \mathrm{D}$ non-consistency of the scans, finding homologous $3 \mathrm{D}$ landmarks is a very challenging task and have therefore approached the problem using a voxel based method. Due to the motion and contrast artifacts corrupting fetal MRI, it is highly desirable to use a similarity measure that is not based on strong assumptions between the two images. We use the normalized mutual information [11] which is: $N M I(X, Y)=\frac{h(X)+h(Y)}{h(X, Y)}$ where $h(X)$ is the marginal entropy of $X$ and $h(X, Y)$ is the joint entropy. Motion correction is performed by aligning globally the low resolution images together and then by aligning every slice of the low resolution images to the reconstructed high resolution image.

Low Resolution Image Registration. The first step consists of approximately globally aligning the $n$ low resolution images. One low resolution image is chosen arbitrarily as the reference image and is used to define a global coordinate system. The other low resolution images are rigidly registered to the reference image (6 degrees of freedom: 3 translations and 3 rotations). The 3D rigid transformation between an image $I_{L R}^{i}$ and the reference image is denoted $T_{1}^{i}$. The high resolution volume coordinate system is chosen to match with the global coordinate system defined previously (see Figure 1).

Slice Registration. Once the low resolution images are roughly globally aligned, slice motion artifacts are corrected by rigidly registering a slice to the current reconstructed high resolution volume. We register the slices of low resolution image $I_{L R}^{i}$ with the volume reconstructed using the $n-1$ other low resolution images $I_{L R}^{j}, j \in\{1 \ldots n, j \neq i\}$. A local coordinate system is defined for every slice and the transformation between a slice and the high resolution volume is composed by two rigid transformations: $\mathbf{y}_{\mathbf{k}}^{\mathbf{i}}=T_{1}^{i} T_{2_{k}}^{i} \mathbf{x}_{\mathbf{k}}^{\mathbf{i}}$ where $T_{2_{k}}^{i}$ denotes the $3 \mathrm{D}$ rigid transformation from the slice $k$ of the low resolution image $i$ to the low resolution image $i$ coordinate system, $\mathbf{x}_{\mathbf{k}}^{\mathbf{i}}$ is the slice pixel coordinates and $\mathbf{y}_{\mathbf{k}}^{\mathbf{i}}$ the voxel coordinates in the compounded high resolution volume (see Figure1).

Optimization. For both image and slice registration, a gradient ascent method is used to maximize the normalized mutual information. Concerning slice registration, we exploit the interleaved acquisition to propose a hierarchical approach

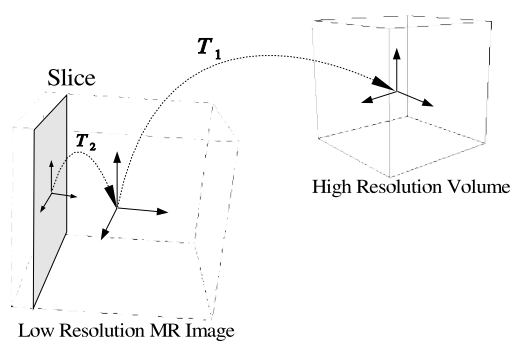

Fig. 1. Coordinate systems used during the slice and image registration 
to provide a starting estimate to the optimization procedure. Slices of a low resolution image are ordered by acquisition time and are separated into two groups. The optimization consists in finding the optimal rigid transformations $T_{2}$ between the new groups of slices and the low resolution image coordinate system. The similarity measure is computed between the current reconstructed volume and the group of slices. This is performed for the $n$ low resolution images. Once the convergence criterion is reached, each group is divided into two groups and the optimization procedure is then performed on these new groups of slices.

For the final phase of alignment, we maximize independently the normalized mutual information between each slice and the current reconstructed high resolution volume. This uses an iterative slice-by-slice scheme, looping over all slices until the sum of the slice-to-volume criteria (called the global convergence criterion) does not increase. For each 2D-3D registration step, a two-level multiresolution strategy is applied to avoid the pitfall of local optima.

\subsection{Volume Reconstruction}

As the volume reconstruction algorithm is required during registration process and contrast correction algorithm, the reconstruction has to be as fast as possible. To deal with the computation time constraint, a local neighborhood approach has been adopted. For each pixel $\mathbf{x}$ in the slices of the original low resolution scans, the $3 \mathrm{D}$ coordinates $\mathbf{y}$ of $\mathbf{x}$ in the high resolution volume are calculated taking into account the results of the image and slice registration. The intensity value of $\mathbf{x}$ is injected into the reconstructed volume using a kernel $f$ centered at $\mathbf{y}$. To approximate the slice profile of the MRI data, we have used a Gaussian kernel ([9]).

\subsection{Contrast Correction}

We employ a contrast correction step to correct the local relative intensity distortion between the low resolution images to allow accurate compounding. Intensity changes from one 2D slice to another are both global and local. To address this issue, one low resolution image is used as a reference for tissue intensities and the other low resolution images are corrected to it. We consider the relationship between contrasts in the low resolution image to be corrected and the reference image as a spatially varying multiplicative field which is assumed to vary smoothly over the field of view. A direct approach to estimation is used where the two volumes are low pass filtered with a Gaussian kernel $\mathcal{G}$. The relative scaling in intensity $\beta_{i}(\mathbf{x})$ at location $\mathbf{x}$ of the low resolution image $I_{L R}^{i}(\mathbf{x})$ is estimated for all slice pixels $\mathbf{x}$ as follows:

$$
\beta_{i}(\mathbf{x})=a_{i} * \frac{\mathcal{G}(I R(\mathbf{x}))}{\mathcal{G}\left(I_{L R}^{i}(\mathbf{x})\right)} \quad \text { with } \quad a_{i}=\frac{\sum_{\mathbf{x}} I_{L R}^{i}(\mathbf{x})}{\sum_{\mathbf{x}} I_{L R}^{i}(\mathbf{x}) \frac{\mathcal{G}(I R(\mathbf{x}))}{\mathcal{G}\left(I_{L R}^{i}(\mathbf{x})\right)}}
$$

where $I R$ is the intensity reference image, $I_{L R}^{i}$ is the low resolution image considered and $a_{i}$ is used to keep the mean value of the image $I_{L R}^{i}$ unchanged. $I R$ is an arbitrary low resolution image projected in the reconstruction space. The corrected low resolution image $\hat{I}_{L R}^{i}$ is then: $\hat{I}_{L R}^{i}(x)=\beta_{i}(x) I_{L R}^{i}(x)$. 


\subsection{Overview of the Algorithm}

The proposed method consists in the following steps:

1. Apply global rigid registration between all the low resolution images.

2. Create initial slice registration estimate by hierarchical application of Slice Registration Algorithm

3. For each Resolution:

(a) Apply Slice Registration Algorithm

(b) Test Global Criteria Reached

4. Apply relative intensity distortion correction

5. Reconstruct the final high resolution volume

Where the slice registration algorithm is the following:

1. For each low resolution image $I_{L R}^{i}$ :

(a) Reconstruct a high resolution volume using the low resolution images $I_{L R}^{j}, j \in\{1 \ldots n, j \neq i\}$

(b) For each slice in $I_{L R}^{i}$, perform the 2D-3D registration.

\section{Material and Evaluation Procedure}

\subsection{Simulated Motions on Real MR Datasets}

In order to perform the most realistic simulation possible, we used as a starting point a 3D MRI acquisition of a premature neonate of similar gestational age (26 weeks gestational age) to the fetal MRI studies, but with high image quality and no motion. The images consisted of a T1 weighted SPGR image with a spatial resolution of $0.7 \times 0.7 \times 2.1 \mathrm{~mm}$. The data were interpolated to $1 \mathrm{~mm}$ isotropic voxels.

Anisotropic subsampled volumes with 6 DOF motion artifact were simulated from the isotropic neonatal image. The resolution of these subsampled volumes is $1 \times 1 \times 3 \mathrm{~mm}$. Motion artifacts were defined by the maximum magnitude of the head motion and by the head motion type. The first simulated motion was a sudden motion. It simulated a short fast movement of the head during a short portion of the acquisition. The second type of motion was a temporally smooth motion simulated using B-Splines. The displacements were chosen from a uniform distribution between $[-\mathrm{x}, \mathrm{x}] \mathrm{mm}$ in each direction and between $[-\mathrm{x}, \mathrm{x}]$ degrees for each rotation, $x \in\{1,5,10\}$.

The accuracy was assessed by computing a registration error measured on a set of 4 points (bounding square) $P_{i}$ distributed within every slices as follows: $R M S=\sqrt{\frac{1}{n} \sum_{i=1}^{n} T R E_{i}^{2}}$, where TRE is the target registration error defined as follows: $T R E_{i}=\left\|P_{i}-\hat{T}^{-1}\left(T^{*}\left(P_{i}\right)\right)\right\|^{2} . T^{*}$ denotes the theoretical transformation and $\hat{T}$ is the estimated geometric transformation.

\subsection{Fetal Datasets}

A typical clinical study of a fetus at our institute follows the following procedure: a quick localizer sequence is obtained in less than 30 seconds during maternal 
free breathing in order to determine the location of the fetal head. No sedation or contrast agents were administered during the study. Conventional Single-shot Fast Spin-echo (SSFSE) T2-weighted images are then acquired during normal maternal breathing (a single slice is acquired in less than one second). Sets of slices were acquired in the axial, sagittal, and coronal planes with respect to the fetal brain, based on the initial localizer. All images were acquired in an interleaved manner to reduce cross-talk between slices. The in plane resolution was about $1 \mathrm{~mm}$ (3mm slice thickness).

\section{Results}

From the simulated motion experiments on premature neonatal data, we evaluated the RMS registration error for four points at the corners of a box within the brain tissue of size $100 \mathrm{~mm} \times 100 \mathrm{~mm}$ for each slice. These are presented in Table1. For all cases the final overall slice alignment error was significantly reduced by the alignment process. Overall, even with the presence of strong motion arte-

Table 1. Accuracy evaluation for the simulated data sets (RMS errors are expressed in $\mathrm{mm}$ )

\begin{tabular}{|c|c|c|c|c|}
\hline Maximum Magnitude & \multicolumn{2}{|c|}{ Sudden Motion } & \multicolumn{2}{|c|}{ Smooth Motion } \\
\hline & RMS start. & $\overline{\mathrm{RMS}}$ end & RMS start. & RMS end \\
\hline \pm 1 & 0.94 & 0.63 & 0.72 & 0.66 \\
\hline \pm 5 & 4.79 & 0.78 & 2.55 & 0.73 \\
\hline \pm 10 & 9.71 & 1.22 & 3.88 & 0.66 \\
\hline
\end{tabular}

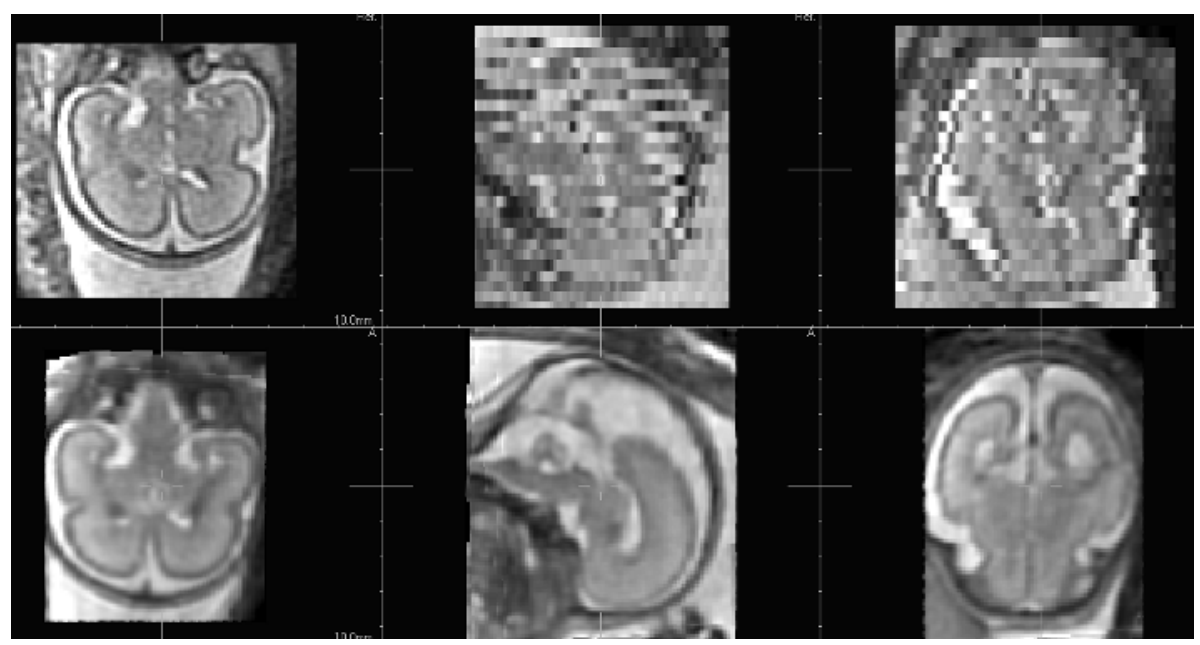

Fig. 2. Subject (a): acquired fetal MR coronal image data (top row) with a resolution of $1 \times 1 \times 3 \mathrm{~mm}$. Lower row shows the resulting reconstructed high resolution image. 


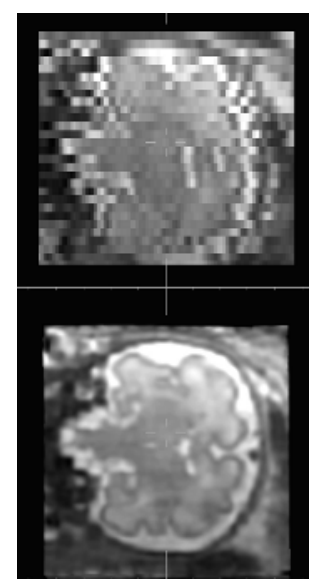

Subject (b)

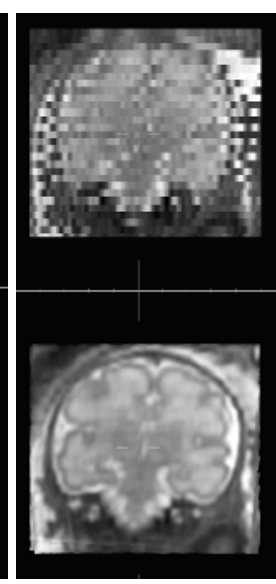

Subject (c)

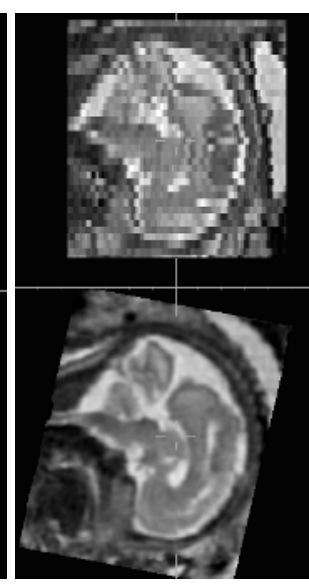

Subject $(d)$

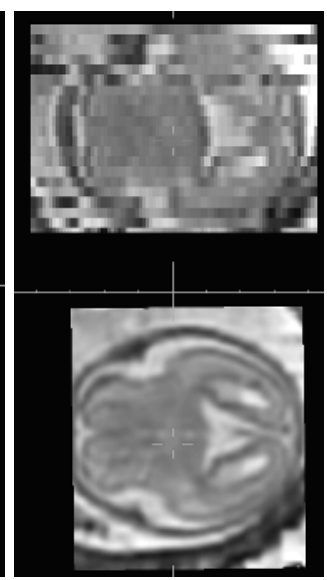

Subject (e)

Fig. 3. Further examples of acquired fetal MR image data (top row) and reconstructed high resolution images (bottom row) from four subject studies

facts, RMS errors remained about the in plane resolution which is $1 \mathrm{~mm}$. In all the cases, visual inspection by clinicians revealed very promising reconstructed image quality.

We then applied the algorithm to clinically acquired fetal MRI datasets using the same optimization parameters. Results are summarized in Figures 2 and 3 . Figure 2 shows one original low resolution coronal image compared to the high resolution reconstructed image for coronal, sagittal and axial views. Figure 3 shows results from four different datasets one original low resolution image and the corresponding high resolution reconstructed image. These figures clearly show the quality improvement in terms of resolution and motion correction providing by the proposed compounding method.

\section{Discussion}

The ability to study the developing fetal brain in high resolution promises to provide a vital source of clinical information which could contribute directly to a number of challenging clinical questions. It permits the application of many quantitative morphometric analysis methods [12,2, developed to study the adult brain to probe the process of normal and abnormal brain development. Critically, high resolution imaging is the key to seeing the process of cortical folding [5, 1, 3], while consistent contrast allows us to study patterns of myelination. Such data is valuable both to specific clinical questions and, more fundamentally, to an improved understanding of the process of human brain development.

In this paper we have described a new methodology to reconstruct in vivo high resolution 3D MRI scans of the fetal brain by proposing to compound multiple sets of orthogonal 2D MRI slices. The method makes use of a novel 
combination of $2 \mathrm{D}$ to $3 \mathrm{D}$ registration, relative inhomogeneity correction and high resolution reconstruction from sparse data. This is achieved by first globally registering the low resolution images, and then applying an iterative slice alignment scheme which seeks to refine the 3D positioning of each slice with respect to the current combined high resolution image. As a final step, a relative intensity correction is applied between the low resolution images to remove the differences in relative signal strength across the different acquisitions in each region of the fetal brain.

Visual inspection of the obtained results on clinical data are very encouraging. The developed algorithm automatically reconstructs a $3 \mathrm{D}$ high resolution geometrically consistent image and it has proved to be robust to large artifacts. This represents an important step towards 3D quantitative analysis of the fetal brain. Further work is needed to better understand the capabilities and limitations of the approach on a range of both fetal anatomies and fetal motion profiles.

This work was supported by a Whitaker foundation award RG-01-0115, a NIH Grant R01-MH65392 and a NIH Biomedical Research Partnership grant, R01-EB0822.

\section{References}

1. Batchelor, P.G. et al. Measures of folding applied to the development of the human fetal brain IEEE Trans. Medical Imaging, 21(8), 953-965, 2002.

2. J. P. Boardman,J.P. et al. An evaluation of deformation-based morphometry in the developing human brain and detection of volumetric changes associated with preterm birth MICCAI, 2003

3. Cachia, A. et al. A Primal Sketch of the Cortex Mean Curvature: a Morphogenesis Based Approach to Study the Variability of the Folding Patterns IEEE Trans. Medical Imaging, 22(6), 754-765, 2003.

4. Coakley, F.V. et al. Fetal MR imaging: A developing modality for the developing patient American Journal of Roentgenology, 182: 243-252, 2004.

5. Inder, T.E. et al. Periventricular white matter injury in the premature infant is followed by reduced cerebral cortical gray matter volume at term Ann Neurol., 46(5), 755-60, 1999.

6. Levine, D. Fetal magnetic resonance imaging Journal of Maternal-Fetal and Neonatal Medicine, 15:85-94, 2004.

7. Lötjönen, J. et al. Correction of Movement Artifacts from 4D Cardiac Short- and Long-Axis MR Data MICCAI, 405-412, 2004.

8. Moore, J. and Drangova, M. and Wierzbicki, M. and Barron, J. and Peters, T. A High Resolution Dynamic Heart Model Based on Averaged MRI Data MICCAI, 549-555, 2003.

9. Ohbuchi, R. and Chen, D. and Fuchs, H. Incremental volume reconstruction and rendering for 3D ultrasound imaging SPIE Visualization in Biomedical Computing, 1808:312-323, 1992.

10. Prayer, D. and Brugger, P. C. and Prayer, L. Fetal MRI: techniques and protocols Pediatr Radiol, 34:685-693, 2004.

11. Studholme, C. and Hill, D.L.G. and Hawkes, D.J. An overlap invariant entropy measure of 3D medical image alignment Pattern Recognition, 32(1):71-86, 1999.

12. Studholme, C. et al. A deformation morphometry study of the influence on the pattern of brain tissue development in premature neonates HBM, 2005. 